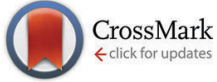

Cite this: Phys. Chem. Chem. Phys., 2015, 17, 23279

DOI: $10.1039 / c 5 c p 90146 d$

www.rsc.org/pccp

\title{
Correction: The strength and directionality of a halogen bond are co-determined by the magnitude and size of the $\sigma$-hole
}

\author{
Michal Kolář, abc Jiří Hostaša and Pavel Hobza*ad
}

Correction for 'The strength and directionality of a halogen bond are co-determined by the magnitude and size of the $\sigma$-hole' by Michal Kolár̆ et al., Phys. Chem. Chem. Phys., 2014, 16, 9987-9996.

Part of the original work ${ }^{1}$ was dedicated to investigation of directionality of halogen bonds. We concluded that the directionality of halogen bonding decreases with the increasing size of the halogen $\sigma$-hole, i.e. with the atomic number of the halogen. We admit that our use of the terminology was not particularly fitting and we aimed at putting our formulations into better order. The numerical results remain unchanged, but here our misuse of the term "directionality" is to be corrected for.

There is experimental evidence that halogen bonds tend to adopt certain geometric patterns with higher propensity than other patterns. This tendency is in common sense understood as directionality. Particularly, the $\mathrm{R}-\mathrm{X} \cdots \mathrm{Y}$ angle, where $\mathrm{X}$ is a halogen, $\mathrm{Y}$ is an electron donor and $\mathrm{R}$ is an arbitrary chemical group or atom, tends to be $180^{\circ}$. This tendency to linearity seems to be higher for heavier halogens than for lighter ones. ${ }^{2-4}$ In other words, the halogen bonds of iodine are more directional than those of bromine or chlorine, which contradicts our previous statements.

We found out that the angular channel allowing the approach of hydrogen fluoride or an argon atom to halogenbenzenes increases with the increasing atomic number of the halogen and that this corresponds to the increase of $\sigma$-hole size (Fig. 1). Consequently, the increasing directionality with the halogen atomic number is rather counterintuitive.
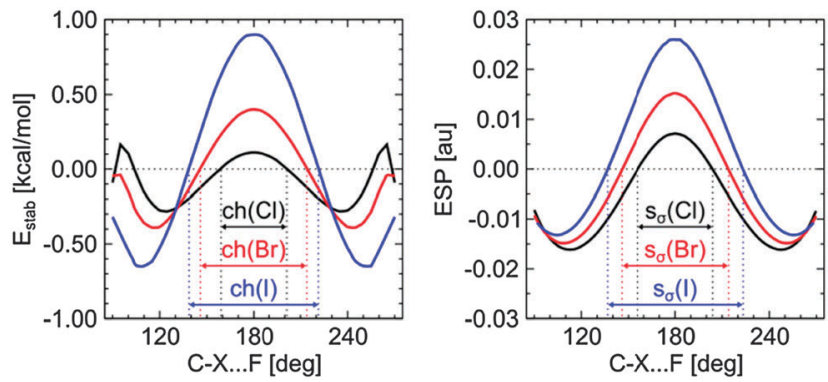

Fig. 1 Left: The angular dependence of the stabilization energy $E_{\text {stab }}$ of hydrogen fluoride with halogenbenzenes calculated at B3LYP-D3/def2-QZVP level. The channel of attractive interaction $c h$ is depicted. Right: The angular dependence of electrostatic potential (ESP) calculated on the surface of 0.001 au electron density. $\sigma$-hole size is labelled as $\mathrm{s}_{\sigma}$.

It is however the energetic penalty brought upon an angular distortion, which determines the directionality. In Fig. 1, left panel, it is shown that the angular profile of stabilization energy is steeper for iodobenzene than for bromo- and chlorobenzenes. This means then that in order to distort the geometry out of $180^{\circ}$ one needs higher energy for an iodinated complex than for a chlorinated one. Similarly, the modulus of the gradient of electrostatic potential (ESP) is larger for heavier halogens, which consequently drives their halogen bonds towards linearity more strongly than for lighter halogens. Thus the directionality of

\footnotetext{
${ }^{a}$ Institute of Organic Chemistry and Biochemistry, Academy of Sciences of the Czech Republic, v. v. i., Flemingovo nam. 2 , 166 10 Prague 6, Czech Republic. E-mail: pavel.hobza@uochb.cas.cz

${ }^{b}$ Computational Biophysics, German Research School for Simulation Sciences GmbH, 52425 Jülich, Germany

${ }^{c}$ Institute for Advanced Simulations (IAS-5), Forschungszentrum Jülich GmbH, D-52425 Jülich, Germany

${ }^{d}$ Regional Centre of Advanced Technologies and Materials, Department of Physical Chemistry, Palacky University, Olomouc, 77146 Olomouc, Czech Republic
} 
halogen bonds is not determined solely by the size of the $\sigma$-hole, but rather by both size and magnitude, and more precisely by their angular variations.

In summary, the following trends can be roughly formulated:

- The magnitude of $\sigma$-hole increases in the order $\mathrm{Cl}<\mathrm{Br}<\mathrm{I}$.

- The size of $\sigma$-hole increases in the order $\mathrm{Cl}<\mathrm{Br}<\mathrm{I}$.

- The channel of attractive interaction increases in the order $\mathrm{Cl}<\mathrm{Br}<\mathrm{I}$.

- The modulus of the gradient of ESP increases in the order $\mathrm{Cl}<\mathrm{Br}<\mathrm{I}$.

- The directionality of halogen bonding increases in the order $\mathrm{Cl}<\mathrm{Br}<\mathrm{I}$.

Let us add a note on hydrogen bonding: in terms of ESP, hydrogen is completely positive in most cases. The angular profiles of stabilization energies of hydrogen-bonded complexes are notably shallower than those of halogens. Hence, the directionality of hydrogen bonds is lower than of halogen bonds. ${ }^{5-7}$

The Royal Society of Chemistry apologises for these errors and any consequent inconvenience to authors and readers.

\section{References}

1 M. Kolář, J. Hostaš and P. Hobza, Phys. Chem. Chem. Phys., 2014, 16, 9987-9996.

2 J. P. Lommerse, A. J. Stone, R. Taylor and F. H. Allen, J. Am. Chem. Soc., 1996, 118, 3108-3116.

3 A. Bauzá, D. Quiñonero, P. M. Deyà and A. Frontera, CrystEngComm, 2013, 15, 3137-3144.

4 P. Auffinger, F. A. Hays, E. Westhof and P. S. Ho, Proc. Natl. Acad. Sci. U. S. A., 2004, 101, 16789-16794.

5 Z. P. Shields, J. S. Murray and P. Politzer, Int. J. Quantum Chem., 2010, 110, 2823-2832.

6 S. Tsuzuki, A. Wakisaka, T. Ono and T. Sonoda, Chem. Eur. J., 2012, 18, 951-960.

7 M. H. Kolář and P. Hobza, Chem. Rev., 2016, in preparation. 